\title{
Phylogenetic structure and habitat associations of Beauveria species isolated from soils in Slovakia
}

\author{
Juraj Medo $^{1 *}$, Jaroslav Michalko ${ }^{2,4}$, Janka Medováa $^{3}$, Ludovít Cagáń $^{1}$ \\ ${ }^{1}$ Slovak University of Agriculture in Nitra, Tr. A. Hlinku 2, 94976 Nitra, Slovakia \\ ${ }^{2}$ Institute of Plant Genetics and Biotechnology, Slovak Academy of Sciences, Akademická 2, 95007 Nitra, \\ Slovakia \\ ${ }^{3}$ Constantine the Philosopher University in Nitra, Tr. A. Hlinku 1,949 74 Nitra, Slovakia \\ ${ }^{4}$ Institute of Forest Ecology, Arboretum Mlynany, Slovak Academy of Sciences, Vieska nad Zitavou 178, 951 \\ 52 Slepcany, Slovakia
}

*corresponding author e-mail juraj.medo@uniag.sk, tel +421 376415810

\begin{abstract}
The phylogenetic structure of 109 soil-borne entomopathogenic Beauveria isolates acquired using the Galleria mellonella bait method from different habitat types in Slovakia was determined by sequence analysis of their ITS and Bloc loci. Three Beauveria species were identified; Beauveria bassiana, B. pseudobassiana and B. brongniartii, represented by 51.4 $\%, 43.1 \%$ and $5.5 \%$ of acquired isolates, respectively, which were resolved into 15, 1 and 1 distinguishable haplotypes. Correlation analysis with the habitat type and individual habitat characteristics showed strong preferences of the most prevalent haplotypes for agricultural $(B$. bassiana A1) and forest habitats (B. pseudobassiana) which has possible implications for conservative biocontrol strategies.
\end{abstract}

Keywords: phylogenetic structure, Beauveria spp., entomopathogenic fungi, habitat preference

\section{Introduction}

Anamorphic entomopathogenic fungi of the genus Beauveria belong to the most commonly isolated facultative pathogens of insect pests (Chandler et al., 1997; Bidochka et al., 1998; Vega et al., 2012). A high level of genetic diversity is commonly detected within natural Beauveria populations. General phylogenetic markers such as intergenic spacer (ITS) or elongation factor 1- $\alpha$ are suitable for distinguishing between Beauveria species but are not 
able to characterize this diversity in deep. Recently developed highly variable markers, specifically the Bloc marker (a nuclear intergenic marker developed for phylogenetic investigation of Beauveria) as well as microsatellites enable further resolution of Beauveria isolates at the level of individual genotypes (Coates et al., 2002; Meyling and Eilenberg, 2006; Castrillo et al., 2007; Hollingsworth et al., 2011). Phylogenetic studies using these markers have shown that diversity within the Beauveria genus could be both on inter- and intra-specific level. For example, it has been found that B. bassiana sensu lato and $B$. brongniartii represent a complex of morphologically cryptic phylogenetic species (Rehner, 2005; Rehner et al., 2006), which led to a revision of Beauveria taxonomy. Three morphologically similar phylogenetic lineages within the $B$. bassiana complex were reclassified as $B$. pseudobassiana (previously designated as Clade C), B. varroae and $B$. kipukae. Additionally, two sister lineages within the B. brongniartii complex were reclassified as B. asiatica and B. australis, which increased the number of currently known Beauveria species to twelve (Rehner et al., 2011). To distinguish between the remaining thus far unresolved lineages within the $B$. bassiana complex a temporary annotation system has been proposed, which designates lineages based on the continent from which they were isolated followed by a digit indicating the order of their discovery, e.g. Eu_1 (Meyling et al., 2009). However, many detected lineages have a worldwide distribution; therefore, an ad hoc naming system based on Rehner and Buckley (2005) without indication of continent should be preferred (e.g. A1), and a reference isolate for each lineage should be deposited in a culture collection (Stephen A. Rehner, personal communication).

Soil is the most commonly examined environment when assessing Beauveria diversity. Baiting techniques using susceptible larvae of Galleria mellonella (Lepidoptera: Pyralidae), commonly referred to as the "Galleria bait method" (GBM), or Tenebrio molitor (Coleoptera: Tenebioidae) are most widely used for isolation of soil-borne Beauveria fungi, although the use of selective media or a combination of both methods may improve the isolation efficacy (Meyling et al., 2009; Medo and Cagáň, 2011). Three species of Beauveria are commonly detected using these methods: B. bassiana, B. pseudobassiana, and $B$. brongniartii. Using the GBM B. bassiana A1 was found by Pérez-González et al. (2014) to be the most common genotype in Mexican agricultural soils. The GBM was also used by Sevim et al. (2010) to find that B. pseudobassiana and B. bassiana A1 and A11 were the most abundant lineages in Turkey. Meyling et al. (2009), using baiting techniques as well as selective media for isolation of fungi from soil, detected lineages A1 (labeled as Eu_1), A3 (Eu_3) and A11 (Eu_6) in their study in Denmark. Lineages A6 (named as Wd-2) and A3 
(Eu_3) were also identified by Garrido-Jurado et al. (2011), who analyzed the EF1- $\alpha$ region of Spanish B. bassiana isolates from culture collection. Additionally, lineages Eu_8 and Eu_9 were reported by these authors. However, Rehner and Buckley (2005) reported much higher diversity of Beauveria isolates from culture collections collected throughout Europe.

Despite improvements made in isolation, taxonomy, and genotyping of Beauveria fungi, major factors determining their genotypic structure at the population level remain poorly understood. Previous studies have linked a particular genetic group of the fungus with its geographical origin (Reay et al., 2010; Sevim et al., 2010; Meyling et al., 2012b; Cai et al., 2013), although some genetic groups have a world-wide distribution. A large number of studies have tried to find an association between a particular genetic group and its insect host (Maurer et al., 1997; Rehner et al., 2006; Reay et al., 2008; Meyling et al., 2012a; Wang et al., 2013). However, only limited evidence has been found to support this association and the general conclusion is that genetic groups in Beauveria spp. do not have any particular host preferences (Meyling et al., 2009). Certain studies have proposed that habitat-related environmental factors constitute the prime selective forces shaping the genetic structure of facultative entomopathogenic fungi (Vanninen, 1996; Bidochka et al., 1998, 2001, 2002; Meyling and Eilenberg, 2007; Quesada-Moraga et al., 2007; Meyling et al., 2009; Medo and Cagán̆, 2011). Despite the existing consensus, few studies have investigated this hypothesis (Bidochka et al., 2002; Meyling et al., 2009). In our study we addressed this question by phylogenetic and correlation analysis of a subset of Beauveria soil isolates from different habitat types across Slovakia isolated using the GBM.

\section{Material and methods}

\subsection{Soil sampling and isolation of fungi}

Soil samples were collected from various locations within Slovakia during the year 2008 (Figure 1A, Supplementary Table S1) and processed as described in Medo and Cagáň (2011). The sampling sites were selected with the aim of maximizing the spectrum of environmental conditions covered (altitude, soil characteristics, vegetation type). The minimum distance between the adjacent sampling sites was $1 \mathrm{~km}$. For each soil sample the habitat type (forest, field, meadow or hedgerow), altitude of the sampling site, type of the vegetation canopy, and basic soil characteristics ( $\mathrm{pH}$, texture) were recorded (Supplementary Table S1). Beauveria 
fungi were isolated from soil samples using the Galleria mellonella baiting technique as described previously (Medo and Cagán̆, 2011) and cultured on Sabouraud dextrose agar (SDA) (Merck KGaA).

\subsection{Genetic analysis}

DNA was extracted from two-week old single spore fungal cultures using the ZR fungal/bacterial DNA extraction kit (Zymo Research Corp. USA). All PCR reaction mixtures contained $200 \mathrm{mM}$ dNTPs, 1x DreamTaq buffer, 0.5 unit DreamTaq DNA polymerase (Life technologies, USA), $0.5 \mathrm{mM}$ of the corresponding primer, and $0.5 \mu \mathrm{l}$ of non-diluted DNA. Cycling conditions were as follows: $95{ }^{\circ} \mathrm{C}$ for $3 \mathrm{~min}$ followed by 35 cycles of $95{ }^{\circ} \mathrm{C}$ for $30 \mathrm{~s}$, annealing at corresponding temperature for each primer pair for $45 \mathrm{~s}, 72{ }^{\circ} \mathrm{C}$ for $90 \mathrm{~s}$, and a final elongation at $72^{\circ} \mathrm{C}$ for $10 \mathrm{~min}$. Primers used for PCR and sequencing of ITS region were ITS1 and ITS4 (White et al., 1990). Primers 5.1F and B822Ldg were used for $B$. pseudobassiana and $5.1 \mathrm{~F}$ and $3.1 \mathrm{R}$ for other Beauveria isolates to amplify the nuclear intergenic region Bloc. Amplification products were sequenced with primers B822Ldg and B22Udg (Rehner et al., 2011).

Sequences were analyzed in an ABI310 genetic analyzer using BigDye 3.1 sequencing chemistry (Life technologies, USA). Acquired sequences were assembled and processed using the Seaview software (Gouy et al., 2010). Alignment was made using MUSCLE (Edgar, 2004). The acquired unique haplotype sequences as well as the reference sequences (for Genbank accessions see Supplementary Table S2) were used for phylogenetic analysis. A phylogenetic tree was constructed using Phy ML (Guindon et al., 2010) with a GTR substitution model, a BioNJ starting tree with the best of NNI and SPR tree searching, and followed by 1000 bootstrap replications.

\subsection{Statistical analyses}

The presence/absence of certain genetic groups between two habitats was evaluated using cross-tabulation followed by the Fisher exact test and the odds ratio was then calculated. The occurrence of genetic groups at different soil $\mathrm{pH}^{\prime} \mathrm{s}$ and altitudes was evaluated using the analysis of variance along with the Tukey HSD test. These statistics were only computed for Beauveria groups with a sufficient number of representatives, i.e. B. pseudobassiana and $B$. bassiana clade A1. All statistical analyses were performed using the Statgraphics XV package (StatPoint Technologies Inc. USA). The estimates of haplotypic diversity were calculated 
according to Stoddart and Taylor (1988) and expressed as the index of haplotypic diversity $(G)$.

\section{Results and discussion}

Maximum likelihood analysis of the combined ITS and Bloc data resolved 109 analyzed Beauveria isolates into 8 phylogenetic clades (Figure 1B) represented by three Beauveria species: B. bassiana, B. pseudobassiana and B. brongniartii containing 56 (54.1 \%), 47 (43.1 $\%)$ and $6(5.5 \%)$ isolates, respectively. Isolates of B. pseudobassiana and B. brongniartii were each represented by a single phylogenetic clade and haplotype while isolates of $B$. bassiana were resolved into six distinct phylogenetic clades (A1, A2, A3, A6, A11 and AX containing respectively 42, 2, 9, 1, 1 and 1 isolates) comprising 15 fungal haplotypes (Figure 1B).

The recognized species composition of Beauveria fungi in Slovakian soils is similar to that reported by other authors in and outside of Europe (Meyling et al., 2009; Sevim et al., 2010; Garrido-Jurado et al., 2011; Pérez-González et al., 2014; Mayerhofer et al., 2015). The genotypic composition of Beauveria spp., on the other side, varies among studies from different geographic areas. Although the comparison of results among studies is not straightforward due to differences in employed protocols for fungal isolation and different genetic markers for isolate genotyping, the composition of main Beauveria lineages appears to be relatively stable across Europe with B. bassiana A1 and B. pseudobassiana being the predominant lineages. Whether this indicates that the less frequent lineages are worse competitors in the soil environment or that their occurrence is restricted to specific environmental conditions remains to be determined.

Within individual habitats, we found that the haplotypic diversity $(G)$ of Beauveria isolates was highest in field soils $(G=5.39)$ and lowest in forests soils $(G=1.53)$. Only three (A1g, A3b and AX) out of 15 identified B. bassiana haplotypes were isolated from forest soils (Figure 2). The abundance of the two most frequent Beauveria clades, B. bassiana A1 and B. pseudobassiana differed significantly among habitats. The odds of finding B. bassiana A1 within field soils was 45.78 times higher than odds to find it within forest soils $(p<0.001)$. A significant difference was also found between meadow and forest $(p<0.001$, odds ratio 476.19), hedgerow and forest ( $p=0.0043$, odds ratio 19.19), and meadow and field habitats ( $\mathrm{p}=0.013$, odds ratio 10.20). In regards to B. pseudobassiana, differences in its occurrence were significant between the forest and field ( $p<0.001$, odds ratio 12.67), forest and meadow 
( $p<0.001$, odds ratio 48.00), and forest and hedgerow habitats ( $p=0.0152$, odds ratio 8.00). $B$. brongniartii was isolated mostly from forest soils (66.6\%); however, it was only represented by 6 isolates. There is a well-known affinity of certain genus/species of entomopathogenic fungi for specific habitats (e.g. Metharizium is common in agricultural soils, while Isaria in forest soils). The differential preference of $B$. pseudobassiana towards natural habitats and $B$. bassiana towards agricultural habitats is in accordance with previous studies (Sevim et al., 2010; Pérez-González et al., 2014). In contrast, Quesada-Moraga et al., 2007 found Spanish isolates of $B$. bassiana morphospecies equivalently common in both natural and cultivated soils, although no genetic discrimination of isolates was made in their study.

To increase our understanding of environmental factors that could underlay Beauveria spp. habitat preferences we evaluated the influence of individual habitat characteristics on the haplotypic structure of Beauveria isolates (Figure 2A). The diversity of Beauveria spp. haplotypes was strongly affected by both the sampling altitude and the soil $\mathrm{pH}$. The highest diversity was found at the lowest altitudes $(<205 \mathrm{~m} ; G=5.36)$ and in more basic soils $(\mathrm{pH}\rangle$ $7.4, G=4.80)$, whereas diversity was lowest at the highest altitudes ( $>400 \mathrm{~m}, \mathrm{G}=3.01)$ and in more acidic soils ( $\mathrm{pH}<6.6, G=2.93$ ) (Figure $2 \mathrm{~B}$ ). Statistically significant differences were found in the occurrence of B. bassiana clade A1 and B. pseudobassiana at different altitudes (301 resp. $387 \mathrm{~m}, p=0.0342$ ) as well as at different soil $\mathrm{pH}$ (7.14 resp. 6.62, $p=0.005$ ). Interestingly, B. bassiana haplotypes $\mathrm{A} 1 \mathrm{~g}$ and $\mathrm{A} 1 \mathrm{~h}$ were found to have inverse preferences in regards to altitude. Soils with loamy texture had much higher Beauveria haplotypic diversity ( $G=6.29)$ than sandy, clay or organic soils $(G=2.63,2.38$ and 1.57 , respectively). The haplotypic diversity of non-forest vegetation types $(G=4.62 \pm 1.33)$ was about three times higher than that observed in forest vegetation $(G=1.54 \pm 0.23)$ (Figure 2B). Similarly, Quesada-Moraga et al. (2007) isolated Beauveria spp. more commonly in slightly basic soils, in soils with lower organic content and at lower altitudes (below $700 \mathrm{~m}$ ).

While all recorded habitat characteristics seem to influence to some extent the haplotypic composition of soil-borne Beauveria spp., their individual importance needs to be tested experimentally as several habitat characteristics tend to be inter-correlated (e.g. the analyzed forest soils had generally lower $\mathrm{pH}$ and occurred at higher altitudes than the field samples and vice versa). In addition, Bidochka et al. (2002) reported association of certain Beauveria and Metarhizium genetic groups with different level of UV irradiation or temperature regions which were not assessed in our study. It is therefore likely that complex relationships among habitat-associated factors influence genotypic composition of Beauveria fungi in nature. Nevertheless, the strong preferences of certain Beauveria phylogenetic groups 
for specific habitats could provide useful information regarding their more targeted usage as biocontrol agents within a particular habitat, especially if their long-term persistence in an environment is desired.

\section{Acknowledgements}

The authors would like to thank Dr. Stephen A. Rehner, USDA-ARS, Beltsville MD, for advices and tips on Beauveria sequencing and phylogeny. We are also thankful to Allwin McDonald for language corrections. This work was financially supported by the national project VEGA 1/0732/14. 


\section{REFERENCES}

Bidochka, M. J., Kamp, A. M., Lavender, T. M., Dekoning, J., De Croos, J. N. A. 2001. Habitat association in two genetic groups of the insect-pathogenic fungus Metarhizium anisopliae: Uncovering cryptic species? Appl. Environ. Microbiol., 67, 3, 1335-1342

Bidochka, M. J., Kasperski, J. E., Wild, G. A. M. 1998. Occurrence of the entomopathogenic fungi Metarhizium anisopliae and Beauveria bassiana in soils from temperate and near-northern habitats. Can. J. Bot., 76, 7, 1198-1204

Bidochka, M. J., Menzies, F. V., Kamp, A. M. 2002. Genetic groups of the insectpathogenic fungus Beauveria bassiana are associated with habitat and thermal growth preferences. Arch. Microbiol., 178, 6, 531-537

Cai, Y., Pu, S., Nie, Y., Rehner, S., Huang, B. 2013. Discrimination of Chinese Beauveria strains by DGGE genotyping and taxonomic identification by sequence analysis of the Bloc nuclear intergenic region. App.l Entomol. Zool., 48, 3, 255-263

Castrillo, L.A., Ugine, T.A., Filotas, M.J., Sanderson, J.P., Vandenberg, J.D., Wraight, S.P. 2007. Molecular characterization and comparative virulence of Beauveria bassiana isolates (Ascomycota: Hypocreales) associated with the greenhouse shore fly, Scatella tenuicosta (Diptera: Ephydridae). Biological Control 45, 154- 162

Chandler, D., Hay, D., Reid, A.P. 1997. Sampling and occurrence of entomopathogenic fungi and nematodes in UK soils. Appl. Soil Ecol. 5, 133-141.

Coates, B.S., Hellmich, R.L., Lewis L.C. 2002. Beauveria bassiana haplotype determination based on nuclear rDNA internal transcribed spacer PCR-RFLP. Mycological Research 106, 1, 40-50.

Edgar, R. C. 2004. MUSCLE: multiple sequence alignment with high accuracy and high throughput. Nucleic acids research, 32, 5, 1792-1797.

Enkerli, J., Widmer, F. 2010. Molecular ecology of fungal entomopathogens: molecular genetic tools and their applications in population and fate studies. Biocontrol, 55, 1, 17-37 Garrido-Jurado, I., Marquez, M., Ortiz-Urquiza, A., Santiago-Alvarez, C., Iturriaga, E., Quesada-Moraga, E., Monte, E., Hermosa, R. 2011. Genetic analyses place most Spanish isolates of Beauveria bassiana in a molecular group with word-wide distribution. BMC Microbiology, 11, 1, 84 
Gouy, M., Guindon, S., Gascuel, O. 2010. SeaView Version 4: A multiplatform graphical user interface for sequence alignment and phylogenetic tree building. Mol. Biol. Evol., 27, $2,221-224$

Guindon, S., Dufayard, J.-F., Lefort, V., Anisimova, M., Hordijk, W., Gascuel, O. 2010. New algorithms and methods to estimate maximum-likelihood phylogenies: Assessing the performance of PhyML 3.0. Syst. Biol., 59, 3, 307-321

Hollingsworth, R. G., Lysy, A. M., \& Matsumoto, T. K. 2011. Preliminary study of genetic variation in Hawaiian isolates of Beauveria bassiana [Hypocreales, Cordycipitaceae]. Journal of invertebrate pathology 106, 3, 422-425.

Maurer, P., Couteaudier, Y., Girard, P. A., Bridge, P. D., Riba, G. 1997. Genetic diversity of Beauveria bassiana and relatedness to host insect range. Mycol Res, 101, 2, 159-164 Mayerhofer, J., Enkerli, J., Zelger, R., Strasser, H. 2015. Biological control of the European cockchafer: persistence of Beauveria brongniartii after long-term applications in the Euroregion Tyrol. BioControl, 60, 5, 617-629.

Medo, J., Cagán̆, L. 2011. Factors affecting the occurrence of entomopathogenic fungi in soils of Slovakia as revealed using two methods. Biol. Control, 59, 2, 200-208

Meyling, N.V., Eilenberg, J., 2006. Isolation and characterisation of Beauveria bassiana isolates from phylloplanes of hedgerow vegetation. Mycological Research 110, 188-195. Meyling, N. V., Eilenberg, J. 2007. Ecology of the entomopathogenic fungi Beauveria bassiana and Metarhizium anisopliae in temperate agroecosystems: potential for conservation biological control. Biol. Control, 43, 2, 145-155

Meyling, N. V., Lübeck, M., Buckley, E. P., Eilenberg, J., Rehner, S. A. 2009. Community composition, host range and genetic structure of the fungal entomopathogen Beauveria in adjoining agricultural and seminatural habitats. Mol. Ecol., 18, 6, 1282-1293 Meyling, N. V., Pilz, C., Keller, S., Widmer, F., Enkerli, J. 2012a. Diversity of Beauveria spp. isolates from pollen beetles Meligethes aeneus in Switzerland. J. Invertebr. Pathol., $109,1,76-82$

Meyling, N. V., Schmidt, N. M., Eilenberg, J. 2012b. Occurrence and diversity of fungal entomopathogens in soils of low and high Arctic Greenland. Polar Biol., 35, 9, 1439-1445 Pérez-González, V. H., Guzmán-Franco, A. W., Alatorre-Rosas, R., Hernández-López, J., Hernández-López, A., Carrillo-Benítez, M. G., Baverstock, J. 2014. Specific diversity of the entomopathogenic fungi Beauveria and Metarhizium in Mexican agricultural soils. $J$. Invertebr. Pathol., 119, 0, 54-61 
Quesada-Moraga, E., Navas-Cortés, J. A., Maranhao, E. A. A., Ortiz-Urquiza, A., Santiago-Álvarez, C. 2007. Factors affecting the occurrence and distribution of entomopathogenic fungi in natural and cultivated soils. Mycol. Res., 111, 8, 947-966

Reay, S. D., Brownbridge, M., Cummings, N. J., Nelson, T. L., Souffre, B., Lignon, C., Glare, T. R. 2008. Isolation and characterization of Beauveria spp. associated with exotic bark beetles in New Zealand Pinus radiata plantation forests. Biol. Control, 46, 3, 484494

Reay, S. D., Brownbridge, M., Gicquel, B., Cummings, N. J., Nelson, T. L. 2010. Isolation and characterization of endophytic Beauveria spp. (Ascomycota: Hypocreales) from Pinus radiata in New Zealand forests. Biol. Control, 54, 1, 52-60

Rehner, S. A. 2005. Phylogenetics of the insect pathogenic genus Beauveria (pp. 3-27). Oxford: Oxford University Press.

Rehner, S., Buckley, E. 2005. A Beauveria phylogeny inferred from nuclear ITS and EF1alpha sequences: evidence for cryptic diversification and links to Cordyceps teleomorphs. Mycologia, 97, $84-98$

Rehner, S. A., Minnis, D., Sung, G.-H., Luangsa-ard, J. J., DeVotto, L., Humber, R. A. 2011. Phylogeny and systematics of the anamorphic, entomopathogenic genus Beauveria. Mycologia, 10-302

Rehner, S. A., Posada, F., Buckley, E. P., Infante, F., Castillo, A., Vega, F. E. 2006. Phylogenetic origins of African and Neotropical Beauveria bassiana s.l. pathogens of the coffee berry borer, Hypothenemus hampei. J. Invertebr. Pathol., 93, 1, 11-21

Sevim, A., Demir, I., Höfte, M., Humber, R. A., Demirbag, Z. 2010. Isolation and characterization of entomopathogenic fungi from hazelnut-growing region of Turkey. Biocontrol, 55, 2, 279-297.

Stoddart, J. A., Taylor, J. F. 1988. Genotypic diversity: estimation and prediction in samples. Genetics 118, 4, 705-11.

Vanninen, I. 1996. Distribution and occurrence of four entomopathogenic fungi in Finland: Effect of geographical location, habitat type and soil type. Mycol. Res., 100, 93101

Vega, F. E., Meyling, N. V., Luangsa-ard, J. J., Blackwell, M. 2012. Chapter 6 - Fungal Entomopathogens. In F. E. Vega H. K. Kaya (Eds.), Insect Pathology (Second Edition) (pp. 171-220). San Diego: Academic Press.

Wang, J.-j., Yang, L., Qiu, X., Liu, Y.-g., Zhou, W., Wan, Y.-J. 2013. Diversity analysis of Beauveria bassiana isolated from infected silkworm in southwest China based on 
molecular data and morphological features of colony. World J. Microbiol. Biotechnol. 29, 7, 1263-1269

White, T., Bruns, T., Lee, S., Taylor, J. 1990. Amplification and direct sequencing of fungal ribosomal RNA genes for phylogenetics. In M. Innis, D. Gelfand, J. Shinsky T. White (Eds.), PCR Protocols: A Guide to Methods and Applications (pp. 315-322): Academic Press. 

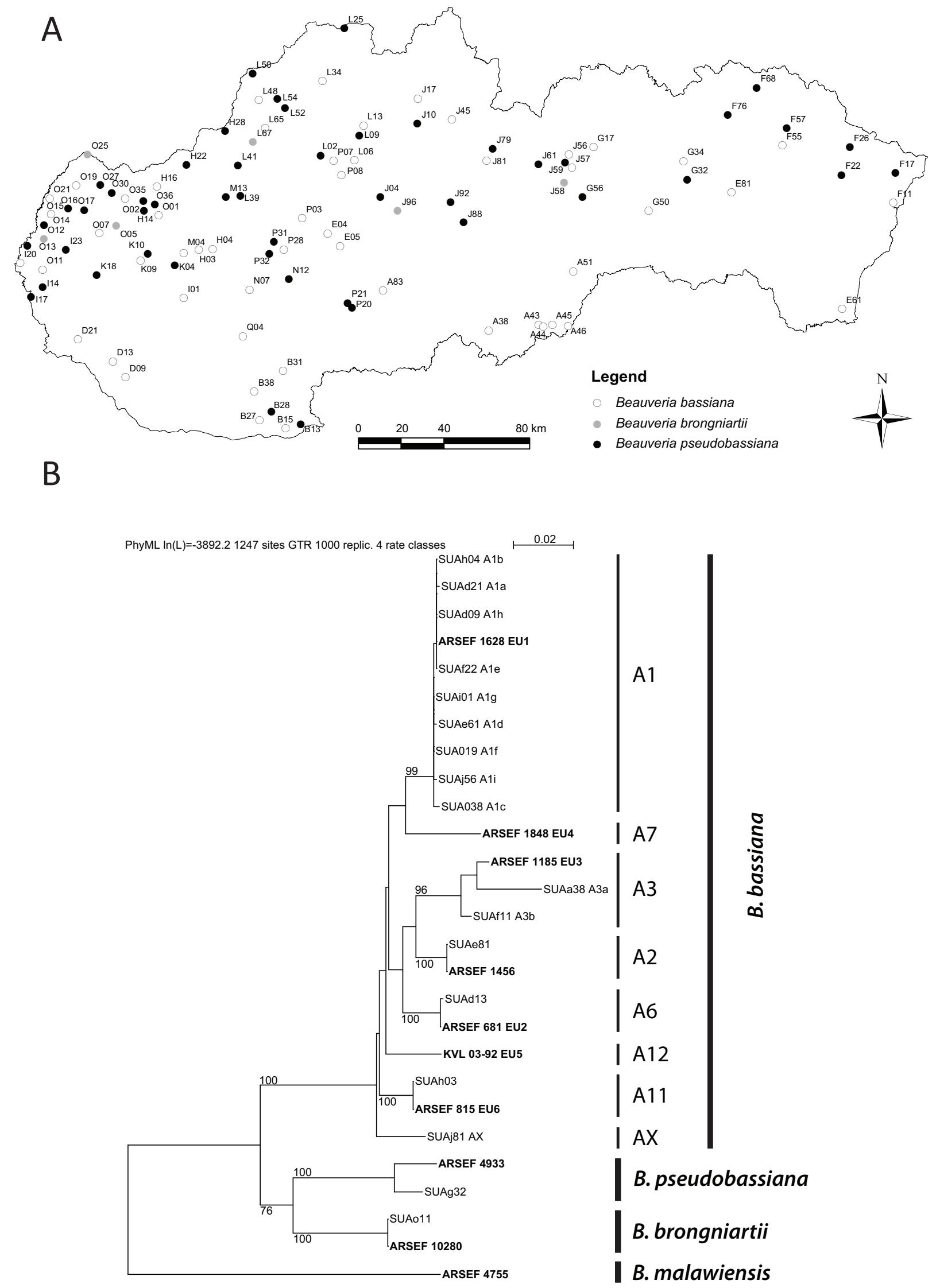


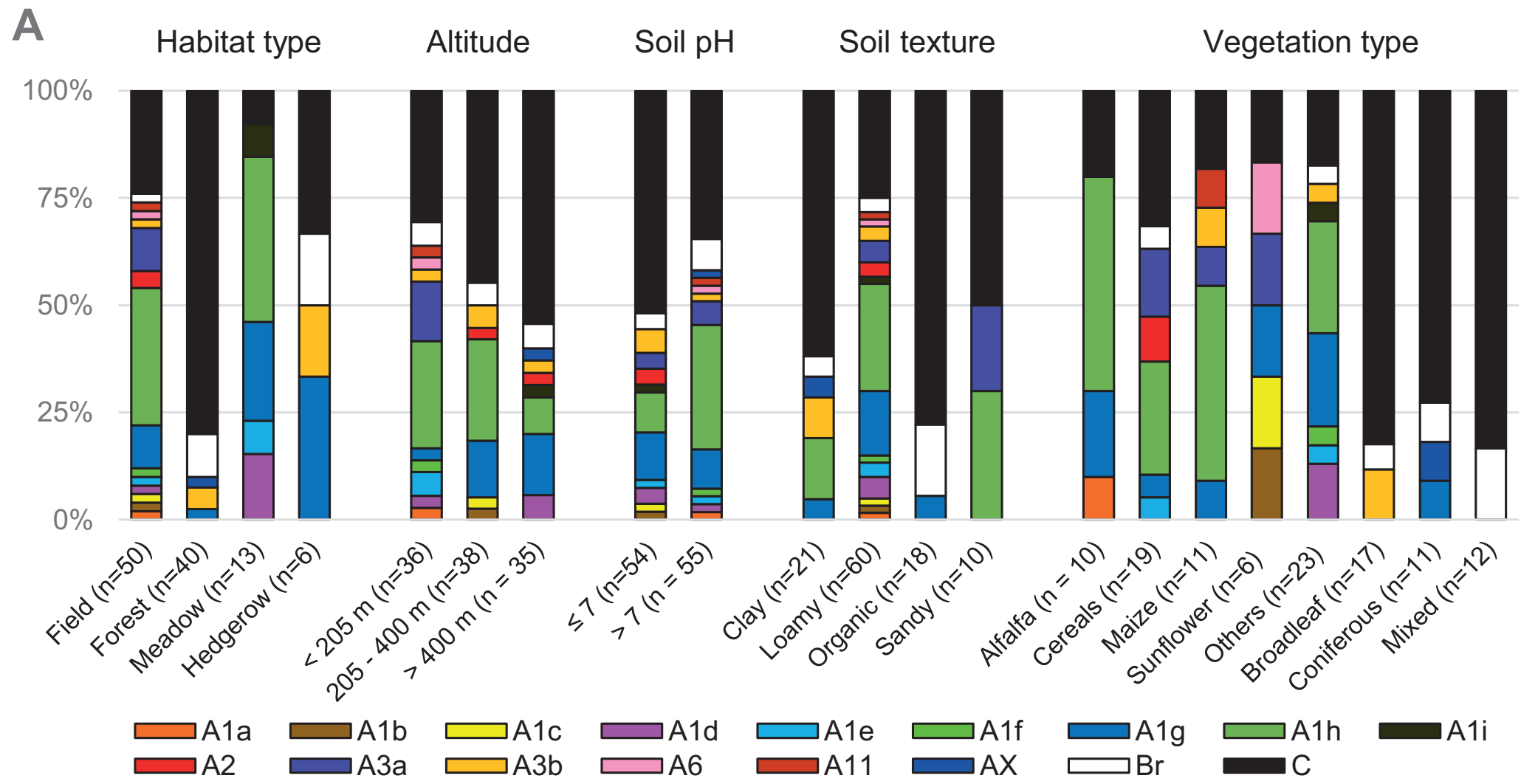

$\begin{array}{llll}\text { Habitat type } & \text { Altitude } & \text { Soil } \mathrm{pH} & \text { Soil texture }\end{array}$

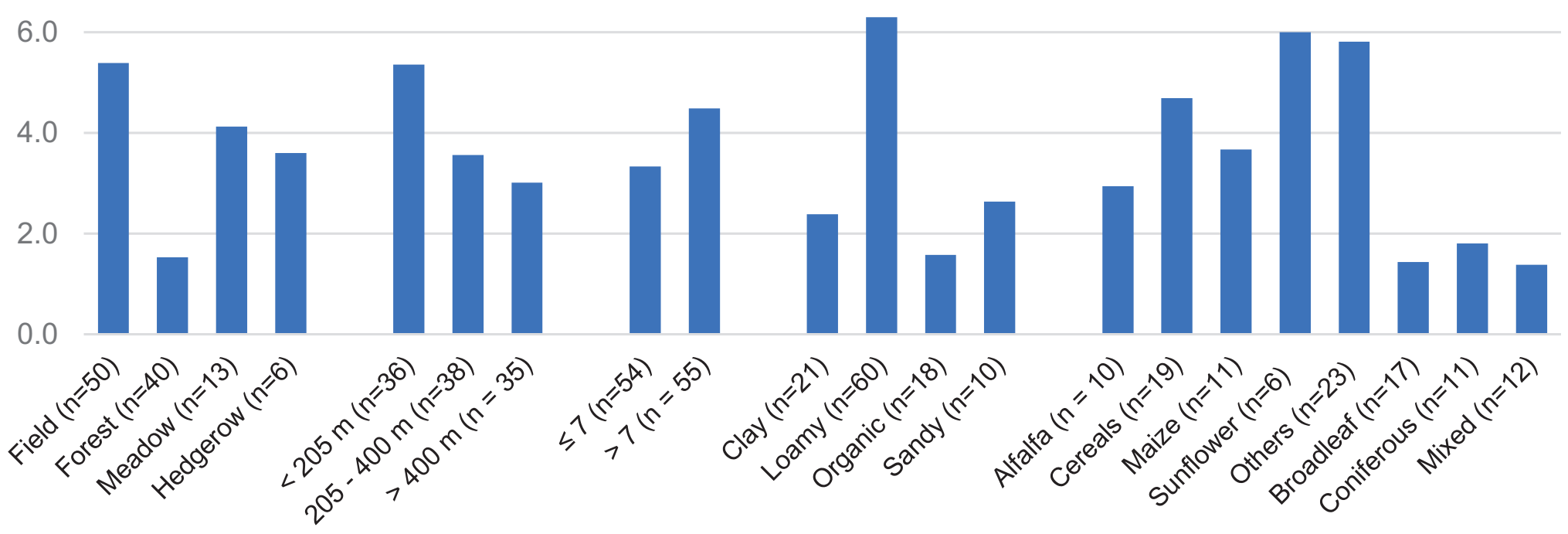

